\title{
Transition from Paris dosimetry system to 3D image-guided planning in interstitial breast brachytherapy
}

\author{
Judyta Wiercińska, MScl,2, Anna Wronczewska, MD, PhD³, Renata Kabacińska, PhD', Prof. Roman Makarewicz, MD²,3 \\ 'Medical Physics Department, Oncology Center, Bydgoszcz, ${ }^{2}$ Chair and Clinic of Oncology and Brachytherapy, Collegium Medicum Bydgoszcz, \\ Nicolaus Copernicus University, Torun, ${ }^{3}$ Brachytherapy Department, Oncology Center, Bydgoszcz, Poland
}

\begin{abstract}
Purpose: The purpose of this study is to evaluate our first experience with 3D image-guided breast brachytherapy and to compare dose distribution parameters between Paris dosimetry system (PDS) and image-based plans.

Material and methods: First 49 breast cancer patients treated with 3D high-dose-rate interstitial brachytherapy as a boost were selected for the study. Every patient underwent computed tomography, and the planning target volume (PTV) and organs at risk (OAR) were outlined. Two treatment plans were created for every patient. First, based on a Paris dosimetry system (PDS), and the second one, imaged-based plan with graphical optimization (OPT). The reference isodose in PDS implants was 85\%, whereas in OPT plans the isodose was chosen to obtain proper target coverage. Dose and volume parameters $\left(\mathrm{D}_{90}, \mathrm{D}_{100}, \mathrm{~V}_{90}, \mathrm{~V}_{100}\right)$, doses at OARs, total reference air kerma (TRAK), and quality assurance parameters: dose nonuniformity ratio (DNR), dose homogeneity index (DHI), and conformity index (COIN) were used for a comparison of both plans.

Results: The mean number of catheters was 7 but the mean for 20 first patients was 5 and almost 9 for the next 29 patients. The mean value of prescribed isodose for OPT plans was $73 \%$. The mean $\mathrm{D}_{90}$ was $88.2 \%$ and $105.8 \%$, the $\mathrm{D}_{100}$ was $59.8 \%$ and $75.7 \%$, the $\mathrm{V}_{\text {PTV90 }}$ was $88.6 \%$ and $98.1 \%$, the $\mathrm{V}_{\text {PTV100 }}$ was $79.9 \%$ and $98.9 \%$, and the TRAK was $0.00375 \mathrm{Gym}^{-1}$ and $0.00439 \mathrm{Gym}^{-1}$ for the PDS and OPT plans, respectively. The mean DNR was 0.29 and 0.42 , the DHI was 0.71 and 0.58 , and the COIN was 0.68 and 0.76 , respectively.

Conclusions: The target coverage in image-guided plans (OPT) was significantly higher than in PDS plans but the dose homogeneity was worse. Also, the value of TRAK increased because of change of prescribing isodose. The learning curve slightly affected our results.

Key words: breast cancer, boost, image-based brachytherapy, Paris dosimetry.

\section{Purpose}

Breast brachytherapy has been used since 1920, when Keynes used radium needles implanted to entire breast [1]. In 1960s, new rules of implantation and dose calculation for interstitial brachytherapy known as Paris dosimetry system (PDS) were established [2]. The PDS became widely used, initially for a low-dose-rate wire sources and later extended to the high-dose-rate applications with ${ }^{192} \mathrm{Ir}$ stepping source [3]. It's role increased when treatment of early-stage breast cancer evolved from radical surgery to breast conserving surgery with adjuvant whole breast irradiation and additional boost dose to the tumor bed $[4,5,6,7]$. The PDS may still be used as a basis for dose specification for volume implants if the recommendations of ICRU report 58 are followed [8]. However, current ad- vances in imaging and technologies applied in brachytherapy showed limitations of PDS [9]. Implementation of 3D image-based brachytherapy planning allows to create treatment plans based on target volumes instead of plans related only to the implant geometry. 3D visualization allows to examine the actual relationship of the implant to the target volume and perform dose-volume analysis of organs at risk (OARs). A variety of dose optimization algorithms enables to obtain required target coverage respecting the required dose homogeneity. Many studies confirmed that increasing the dose to surroundings of the lumpectomy cavity reduced local recurrence rate, but on the other hand, the cosmetic outcome is inversely related to radiation dose [10]. Proper target coverage, high dose homogeneity and conformity, and controlling dose to OARs, therefore, are essential in brachytherapy planning. 
3D imaging in currently available planning software's gives us possibility to evaluate these parameters in clinical cases.

The purpose of this study is to analyze our first experience with 3D image-guided breast brachytherapy, compare dose distribution parameters between PDS, and image based treatment plans as well as to evaluate changes in planning procedure after implementing $3 \mathrm{D}$ system.

\section{Material and methods}

First, 49 consecutive patients during breast conserving therapy treated with 3D image-based high-dose-rate (HDR) interstitial brachytherapy as a boost after external beam radiotherapy were selected for the study. It was our first experience with planning based on computed tomography $(\mathrm{CT})$, and the first couple of patients were implanted only based on surgery scar, clinical examination, and the results of mammography. Rest of them were implanted based on CT created for EBRT planning. Localization of tumor bed was done considering surgical clips. In our hospital, in majority of cases, only one surgery clip is placed on the muscle beneath the tumor bed. On this pre-planning CT we've measured distances in all three dimensions from surgery clips to distinctive elements of patient's body, such as nipple, sternum, or rib. Then a printout containing $3 \mathrm{D}$ visualization of patient's body with all these structures and measurements was prepared. Figure 1 presents an example of the print- out. Distances from this visualization were measured on patient's breast just before implantation to help localize lumpectomy cavity.

During the interstitial brachytherapy procedure, 4 to 13 metal needles in 1, 2 or 3 planes ( 2 patients -1 plane, 31 patients -2 planes, 16 patients -3 planes) were inserted into tumor bed with template guidance. All patients underwent $\mathrm{CT}$ with a slice thickness of $2 \mathrm{~mm}$. The CT images were sent to the brachytherapy planning system Oncentra Masterplan (version 4.3, Nucletron, an Elekta company, Elekta $\mathrm{AB}$, Stockholm, Sweden), where planning target volume (PTV) and OARs (skin, most exposed rib, and ipsilateral lung) were contoured. Two treatment plans were made for every patient. First, based on PDS, using geometrical optimization, and the second one, image-based plan using graphical optimization (OPT). The reference isodose in PDS was $85 \%$ in every case, whereas in OPT plans an isodose was chosen to obtain proper target coverage. Dose and volume parameters $\left(\mathrm{D}_{90}, \mathrm{D}_{100}, \mathrm{~V}_{\text {PTV90, }}\right.$

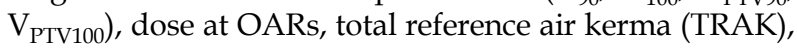
and quality assurance parameters: dose nonuniformity ratio (DNR), dose homogeneity index (DHI), and conformity index (COIN) were used for a comparison of both plans. The following definitions are used in our study [2]:

$$
\mathrm{DNR}=\mathrm{V}_{150} / \mathrm{V}_{100} ;
$$

$$
\begin{gathered}
\text { DHI inside PTV }=\left(\mathrm{V}_{\text {PTV100 }}-\mathrm{V}_{\text {PTV150 }}\right) / \mathrm{V}_{\text {PTV100; }} \\
\text { COIN }=\left(\mathrm{V}_{\text {PTV100 }} / \mathrm{V}_{\text {PTV }}\right) \times\left(\mathrm{V}_{\text {PTV100 }} / \mathrm{V}_{100}\right)
\end{gathered}
$$

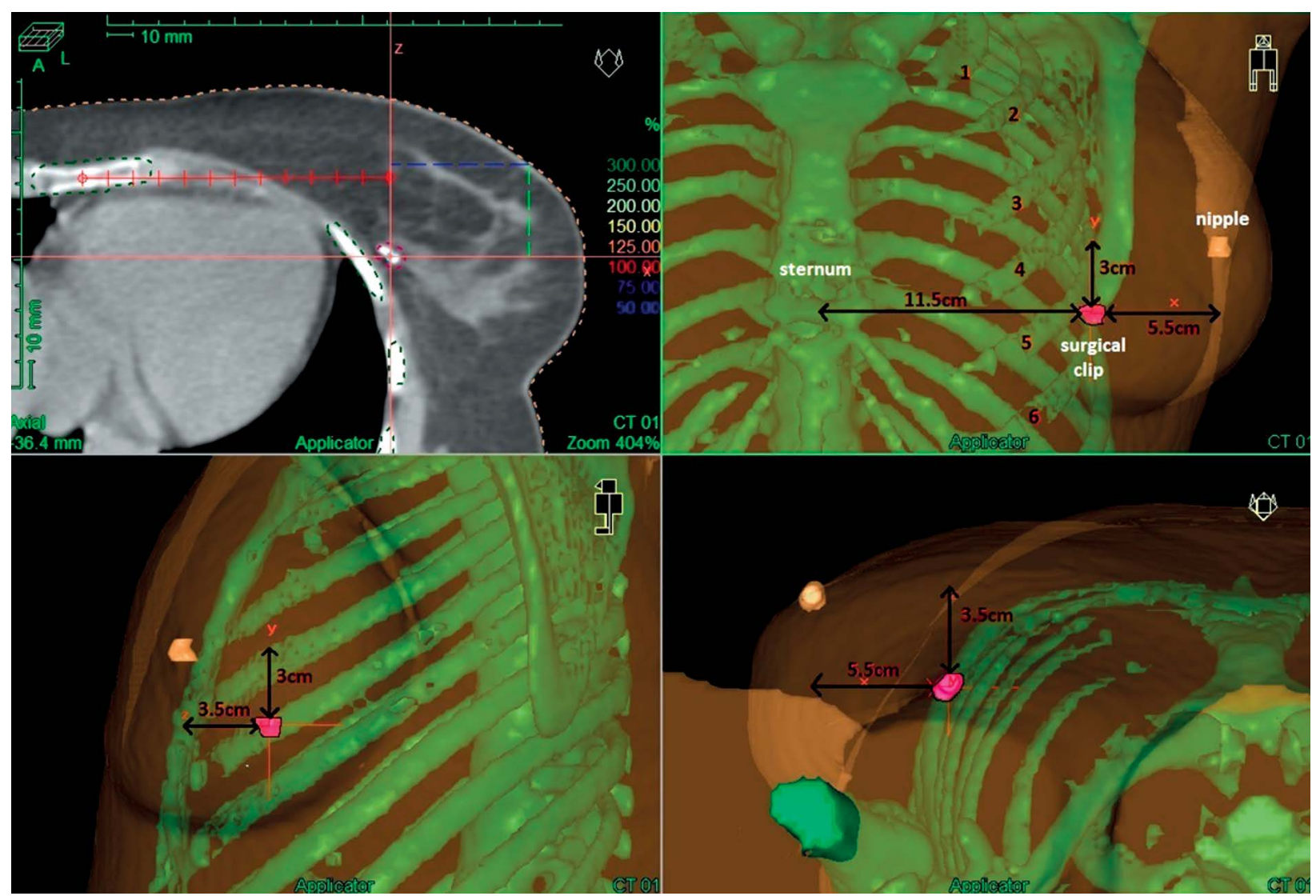

Fig. 1. Printout of pre-planning procedure with ribs counted and numbered and distances marked in all three dimensions from surgery clips to distinctive elements of patient's body (sternum and nipple) 
where $V_{100}$ and $V_{150}$ means volume receiving $100 \%$ and $150 \%$ of prescribed dose, respectively, $V_{P T V 100}$ and $V_{P T V 150}$ means volume of PTV receiving $100 \%$ and $150 \%$ of prescribed dose, respectively, and $V_{P T V}$ means volume of PTV. Doses at OARs were evaluated by a dose at most exposed 0.1cc $\left(\mathrm{D}_{0.1 \mathrm{cc}}\right)$ and at the most exposed 2cc $\left(\mathrm{D}_{2 \mathrm{cc}}\right)$ of specific OAR. The second comparison was done to analyze a learning curve during our first experience with $3 \mathrm{D}$ image-guided planning. All plans (both PDS and OPT) were divided into two groups: group of the first 20 patients and group of the next 29 patients out of all 49 analyzed cases. The comparison was done between dose and volume parameters and quality assurance parameters of both groups. Calculations were performed with Statistica 8.0 (SPSS Inc., Chicago, IL, USA). A difference between two variables was considered statistically significant when the $p$-value was less than or equal than 0.05 .

\section{Results}

One-, two-, and three-plane implants were used. The mean number of catheters was 7 but the mean for the first 20 patients was 5 and almost 9 for the remaining 29 patients. The volume of PTV ranged from $13.6 \mathrm{~cm}^{3}$ to $163.4 \mathrm{~cm}^{3}$ (mean: $58.2 \mathrm{~cm}^{3}$ ), while the mean volume irradiated by prescribed dose was $53.0 \mathrm{~cm}^{3}$ and $66.0 \mathrm{~cm}^{3}$ for PDS and OPT plan, respectively. The mean value of isodose selected for prescribing dose for OPT plans was $73 \%$ in order to achieve acceptable dose coverage. The mean $\mathrm{D}_{90}$ was $88.2 \%$ and $105.9 \%$, the $\mathrm{D}_{100}$ was $59.8 \%$ and $75.7 \%$, the $\mathrm{V}_{\text {PTV90 }}$ was $88.6 \%$ and $98.1 \%$, the $\mathrm{V}_{\text {PTV100 }}$ was $79.9 \%$ and $98.9 \%$, and the TRAK was $0.00375 \mathrm{Gym}^{-1}$ and $0.00439 \mathrm{Gym}^{-1}$ for the PDS and OPT plans, respectively. Doses received by organs at risk were slightly higher in OPT plans because of lower value of prescribed isodose in these plans and amounted to $73.9 \%$ for skin $\mathrm{D}_{0.1 \mathrm{cc}}$ $59.2 \%$ for skin $\mathrm{D}_{2 \mathrm{cc}}, 28.1 \%$ for the most exposed rib, and $21.0 \%$ for ipsilateral lung. The same doses for PDS plans amounted to $67.5 \%$ for skin $\mathrm{D}_{0.1 \mathrm{cc}} 52.8 \%$ for skin $\mathrm{D}_{2 \mathrm{cc}}$ $24.5 \%$ for the most exposed rib, and $18.1 \%$ for ipsilateral lung. All the doses are presented as a percentage of prescribed dose. Better target coverage resulted also in worse homogeneity. We obtained mean DNR of 0.29 and 0.42 , mean DHI inside PTV of 0.71 , and 0.54 for PDS and OPT
Table 1. Mean values of dosimetric parameters and doses at organs at risk (OARs) of both plans

\begin{tabular}{|c|c|c|c|}
\hline $\begin{array}{l}\text { Dosimetric } \\
\text { parameter }\end{array}$ & PDS plan & OPT plan & $p$ value \\
\hline V PTV90 $_{\text {P }}[\%]$ & $88.6 \pm 7.0$ & $98.1 \pm 2.4$ & $<0.05$ \\
\hline$V_{\text {PTV100 }}[\%]$ & 79.9 & .6 & $<0.05$ \\
\hline $\mathrm{D}_{90}[\%]$ & 88.2 & $105.9 \pm 9.5$ & $<0.05$ \\
\hline $\mathrm{D}_{100}[\%]$ & $59.8 \pm 9.4$ & $75.7 \pm 5.4$ & $<0.05$ \\
\hline Skin $D_{0.1 c c}[\%]$ & $67.5 \pm 11.6$ & $73.9 \pm 10.0$ & $<0.05$ \\
\hline Skin $D_{2 c c}[\%]$ & $52.8 \pm 7.4$ & $59.2 \pm 7.7$ & $<0.05$ \\
\hline $\operatorname{Rib} D_{0.1 c c}[\%]$ & $24.5 \pm 17.6$ & $28.1 \pm 17.0$ & $<0.05$ \\
\hline Lung $\mathrm{D}_{2 \mathrm{cc}}[\%]$ & $18.1 \pm 11.2$ & $21.0 \pm 11.6$ & $<0.05$ \\
\hline DNR & $0.29 \pm 0.05$ & $0.42 \pm 0.07$ & $<0.05$ \\
\hline DHI inside PTV & $0.71 \pm 0.05$ & $0.54 \pm 0.09$ & $<0.05$ \\
\hline COIN & $0.69 \pm 0.10$ & $0.76 \pm 0.06$ & $<0.05$ \\
\hline TRAK [Gym $\left.{ }^{-1}\right]$ & $0.0038 \pm 0.0014$ & $0.0044 \pm 0.0017$ & $<0.05$ \\
\hline
\end{tabular}

$V_{\text {PTV9o }}[\%], V_{\text {PTV100 }}[\%]$ - relative percentage of planning target volume, which receives $90 \%$ and $100 \%$ of prescribed dose, respectively; $D_{90}[\%], D_{100}[\%]$ - dose to the $90 \%$ and $100 \%$ volume of interest relative to the prescribed dose, respec tively; $D_{0.1 c c}, D_{2 c c}$-dose at the most exposed $0.1 c c$ and $2 c c$ of volume of interest, respectively, DNR - dose nonuniformity ratio, DHI inside PTV - dose homogeneity index inside planning target volume, COIN - conformity index, TRAK [Gym-1] - total reference air kerma, PDS - Paris dosimetry system, OPT - image-based plan using graphical optimization

plans, respectively. Simultaneously, the optimization of a plan improved our conformity. Mean COIN for PDS plans was 0.69 while for OPT plans it was 0.76. A comparison was made between mean values of all doses and dosimetric parameters of both types of plan. Differences between all these parameters were statistically significant. Mean values of dosimetric parameters and doses at OARs of both plans are shown in Table 1.

During this study, we've observed our learning curve. Most of the parameters had better values in the last 29 patients compared to the first 20 . Table 2 contains mean values of dosimetric parameters divided into these two groups.

Table 2. Mean values of dosimetric parameters divided into two groups of consecutive patients

\begin{tabular}{lcccc}
\multirow{2}{*}{ Dosimetric parameter } & \multicolumn{2}{c}{ PDS plans } & \multicolumn{2}{c}{ OPT plans } \\
\cline { 2 - 5 } & First 20 patients & Next 29 patients & First 20 patients & Next 29 patients \\
\hline$V_{\text {PTV100 }}[\%]$ & 79.5 & 80.3 & 93.5 & 94.2 \\
\hline$D_{90}[\%]$ & 88.8 & 87.8 & 105.0 & 106.4 \\
\hline$D_{100}[\%]$ & 62.0 & 58.3 & 76.7 & 76.0 \\
\hline DNR & 0.33 & 0.26 & 0.44 & 0.4 \\
\hline DHI inside PTV & 0.68 & 0.74 & 0.54 & 0.55 \\
\hline COIN & 0.69 & 0.67 & 0.75 & 0.76
\end{tabular}

$V_{\text {PTV100 }}[\%]$ - relative percentage of planning target volume, which receives $100 \%$ of prescribed dose, $D_{90}[\%], D_{100}[\%]-$ dose to the $90 \%$ and $100 \%$ volume of interest relative to the prescribed dose, respectively, DNR - dose nonuniformity ratio, DHI inside PTV - dose homogeneity index inside planning target volume, COIN - conformity index, PDS - Paris dosimetry system, OPT - image-based plan using graphical optimization 


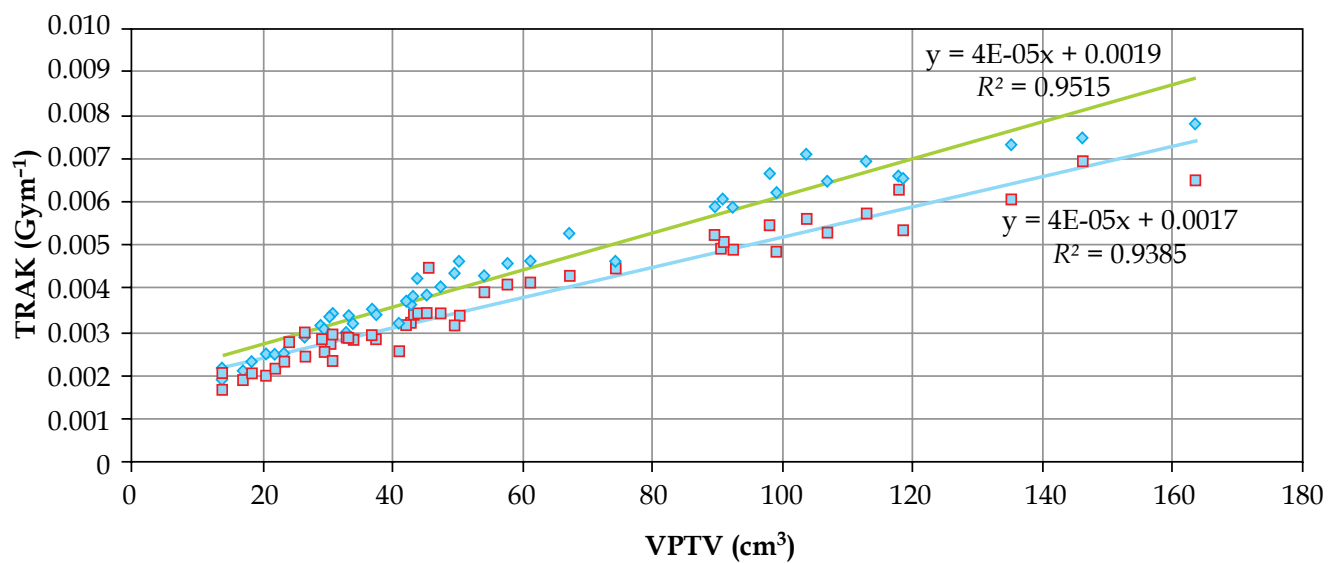

Fig. 2. Correlation between total reference air kerma (TRAK) values for Paris dosimetry system (PDS) (orange) and graphical optimization (OPT) (blue) plans, and volume of planning target volume (PTV)

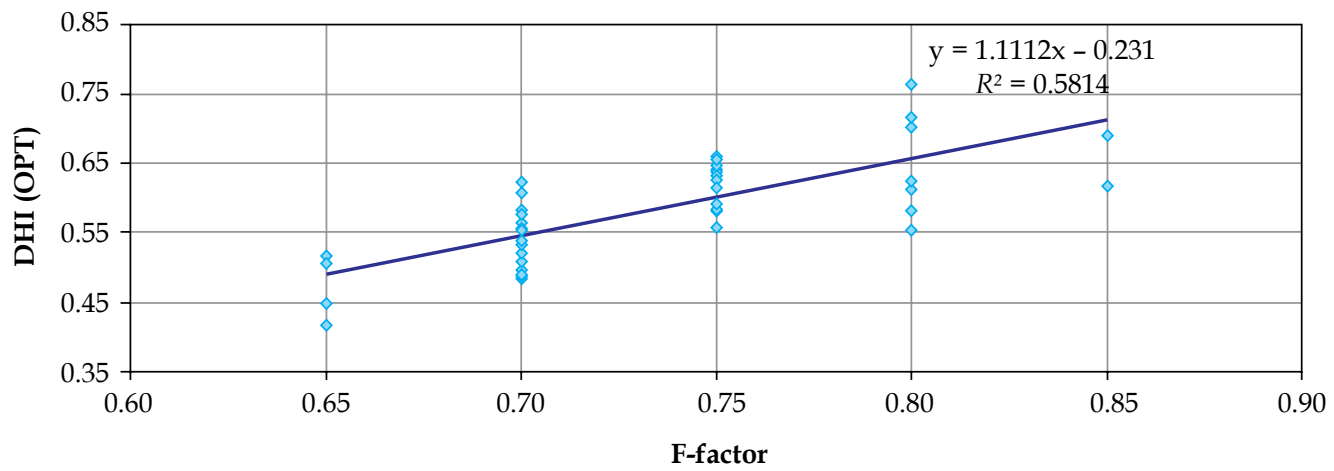

Fig. 3. Correlation between dose homogeneity index for graphical optimization (OPT) plans and value of prescribed isodose (F-factor) chosen for dose specification in these plans

In our study, there was a high linear correlation between TRAKs and volume of PTV (PDS: $\mathrm{R}=0.97$ and OPT: $R=0.98$ ), and also with number of needles (PDS: $\mathrm{R}=0.88$ and OPT: $\mathrm{R}=0.86$ ) for both types of plans. Figure 2 shows correlation between TRAKs and volume of PTV. There was also high linear correlation between TRAK and DHI in PDS plan $(\mathrm{R}=0.8)$ but there were only very poor linear correlations between these parameters in OPT plans $(\mathrm{R}=0.1)$, as well as between TRAK and the value of prescribed isodose $-\mathrm{F}$-factor $(\mathrm{R}=-0.21)$. TRAK depends mostly on volume of PTV, while DHI and value of isodose chosen to obtain proper target coverage depends on implant quality. This results probably in such poor correlations between these factors. There were also high linear correlations between F-factor and DHI $(\mathrm{R}=0.76)$ showed in Figure 3 but there was no linear correlation between F-factor and target coverage in OPT plans $(\mathrm{R}=0.1)$.

\section{Discussion}

Paris dosimetry system has been successfully used for decades in interstitial brachytherapy due to its biggest advantage - homogenous dose distribution. In our study, we implemented standard PDS with geometrical optimization in plans made on CT's and compared to second plans optimized to achieve proper target coverage. The target coverage in image-guided plans (OPT) was significantly higher than in plans based on PDS but the dose homogeneity was worse. Major et al. compared four different plans for interstitial breast brachytherapy [11]. One of them was based on the same principles as our PDS plan, that means standard PDS with geometrical optimization. They pointed high homogeneity of this type of plan with DNR of 0.25 and DHI inside PTV of 0.74 . These values are almost the same as our results $(0.29$ and 0.71 , respectively) but we received better target coverage and dose conformity: $\mathrm{D}_{90}$ was $88 \%$ and $60 \%$, and COIN 0.66 and 0.55 in our study and Major's, respectively. In their another study, Major et al. used the same method of optimization as our OPT plans [12]. Their mean prescribed isodose was $74 \%$, what is very similar to our $73 \%$. They obtained homogeneity parameters slightly better than ours (mean DNR was 0.32 and mean DHI inside PTV was 0.66 , compared to our 0.42 and 0.54 , respectively) but they used pre- and postimplant CT, what probably resulted in better implant. Aristei et al. also used preimplant CT and obtained excellent results of mean $\mathrm{D}_{90}-96.5 \%$ and DHI for the whole implant of 0.76 [13]. Vicini et al. analyzed non-CT-based implantation with geometrical optimized plan and they obtained worse target coverage but very high homogeneity with mean values of $\mathrm{D}_{90}, \mathrm{D}_{100}$, and DHI for whole implant, respectively, $73 \%, 68 \%$, and 0.89 for five selected patients (from 8 selected for the study) [14]. Kestin et al. analyzed retrospectively a group of 11 patients and showed that even slight dwell time adjustment could result in better target coverage from $85.3 \%$ to 
$97.0 \%$ for lumpectomy cavity, while keeping DHI on high level of 0.86 (0.89 before adjustment) [15]. In their study, there was no information about improvement of coverage of entire target volume (cavity with $1 \mathrm{~cm}$ margin). Das et al. obtained excellent results of median $\mathrm{D}_{100}$ of $96 \%$ and mean DHI of 0.73 using geometrical and graphical optimization in CT-based plans [16] but it has to be added that they identified PTV by injecting contrast material inside lumpectomy cavity and used preimplant mammography. Wazer et al. showed that high values of dose homogeneity index reduce risk of late fibrosis, so it is very important to improve homogeneity while keeping good target coverage and conformity of our plans [17]. Major et al. found high improvement of their results after using preimplant CT ( $\mathrm{V}_{\text {PTV100 }}$ from $70 \%$ to $91 \%$, DNR from 0.35 to 0.33 and COIN from 0.40 to 0.68$)[11,18]$. Cholewka et al. came to the same conclusion after comparing their 2D plans without pre-planning to CT based guided plans [19]. They obtained an improvement of $\mathrm{V}_{\text {PTV100 }}$ from $86.1 \%$ to $91.7 \%$ and dose homogeneity index from 0.53 to 0.60 . The same trend could be seen in our study when comparing our first 20 patients implanted mostly basing on surgical scar and clinical examination with the next 29 with pre-planning based on CT scans. We've noticed an increase of $\mathrm{V}_{\text {PTV100 }}$ from $93.5 \%$ to $94.2 \%$ and dose homogeneity index from 0.56 to 0.60 for the whole implant, and from 0.54 to 0.55 for PTV only. It means that further improvements of implant quality could be achieved during the implantation of needles. Tang et al. analyzed impact of implantation method on the implant quality comparing free hand technique under ultrasound guidance with template method based on CT images with radiographic contrast injected into tumor bed before needles insertion [20]. They obtained excellent dose homogeneity index for both methods ( 0.74 for free hand and 0.76 for template technique), with significantly better target coverage and less high doses volumes for insertion with template guidance. There is a number of studies focused on the impact of different image-based pre-planning methods on treatment plan quality $[1,2,4,5$, $6,20,21,22,23]$. All of them confirmed that before implantation as much information as possible should be included to precisely localize the tumor bed.

Slight improvement of our dosimetric parameters could also result from a learning curve. It's too small group and too short period of time to make a reliable evaluation, but a trend could be seen when comparing groups of the first 20 and the next 29 patients. Cholewka et al. analyzed their learning curve in first experiences with 3D image-based partially breast brachytherapy [24]. They've noticed a gentle progress every 6 months during the whole 4 years of observation. The scale of their improvement is similar to our results when comparing the same period of time.

There are very few studies containing information about values of TRAK in breast brachytherapy planning, even it is recommended for reporting in every brachytherapy application by ESTRO [25]. We consider TRAK as very valuable factor, especially in case of transition from standard 2D planning to 3D image-based method. In our study, we noticed $16 \%$ increase of TRAK from $0.0038 \mathrm{Gym}^{-1}$ for standard Paris system based planning to $0.0044 \mathrm{Gym}^{-1}$ for $3 \mathrm{D}$ planning. The reason for that is the lower prescription isodose line what increases irradiated volume. In both types of our planning methods, values of TRAK were highly correlated with volume of PTV as evident from the Graph 1. Major et al. noted the same correlation in their study [18]. Contemporary standards of brachytherapy planning are image-based and all recommendations emphasized that $3 \mathrm{D}$ imaging is crucial to receive proper target coverage [26, 27]. However, longterm studies proved good results after BCS obtained with boost based on PDS. Retrospectives studies provided by Resch et al. on large cohort of 410 women with early stage breast cancer after BCT with interstitial brachytherapy boost showed excellent results with only 16 recurrences ( 2 and 3.9\% in 5-year and 10-year actuarial recurrence rate), and only $7 \%$ of cosmetic outcomes classified as bad [28]. Similar results were obtained by Polgar et al. with $7 \%$ ipsilateral breast failure after median follow-up time of 94 months on cohort of 100 patients [29]. It will take time to collect as many reliable image-guided long-term studies as 2D-based ones available at present.

\section{Conclusion}

Our first experience with 3D image-based planning showed that optimized plans based on CT could significantly improve target coverage and dose conformity but only at the cost of worse dose homogeneity compared to plans based on principles of PDS. We've observed a learning curve during implantation as well as in treatment planning procedure. Implementation of $3 \mathrm{D}$ imaging in pre-planning procedure could result in further improvements of implant quality and allow to obtain satisfactory dosimetric parameters.

\section{Disclosure}

Authors report no conflict of interest.

\section{References}

1. Nag S, Kuske R, Vicini F et al. Brachytherapy in treatment of breast cancer. Oncology(Williston Park) 2001; 15: 195-202.

2. Strnad V, Potter R, Kovacs G. Practical Handbook of Brachytherapy. UNI-MED, Verlag AG, Bremen 2014.

3. Mould RF, Battermann JJ, Martinez AA et al. Brachytherapy from radium to optimization. Nucletron International, Veenendall 1994; 319-330.

4. Luini A, Gatti G, Zurrida S et al. The evolution of the conservative approach to breast cancer. Breast 2007; 16: 120-129.

5. Fijuth J. Brachytherapy in breast cancer. J Contemp Brachytherapy 2009; 1: 117-120.

6. Hepel JT, Wazer DE. A comparison of brachytherapy techniques for partial breast irradiation. Brachytherapy 2012; 11: 163-175.

7. Skowronek J, Chicheł A. Brachytherapy in breast cancer: an effective alternative. Prz Menopauzalny 2014; 13: 48-55.

8. ICRU: Report 58: Dose and volume specifications for reporting Interstitial Therapy. ICRU, Bethseda 1997.

9. Hennequin C, Mazeron JJ, Chotin G. How to use Paris system in the year 2001? Radiother Oncol 2001; 58: 5-6.

10. Van Limbergen E. Indications and technical aspects of brachytherapy in breast conserving treatment of breast cancer. Cancer Radiother 2003; 7: 107-120.

11. Major T, Fodor J, Takácsi-Nagy Z et al. Evaluation of HDR interstitial breast implants planned by conventional and op- 
timized CT-based dosimetry systems with respects to dose homogeneity and conformality. Strahlenther Onkol 2005; 181: 89-96.

12. Major T, Fröhlich G, Polgar C. Assessment of dose homogeneity in conformal interstitial breast brachytherapy with special respect to ICRU recommendations. J Contemp Brachytherapy 2011; 3: 150-155.

13. Aristhei C, Tarducci R, Palumbo I et al. Computed tomography for excision cavity localization and 3D-treatment planning in partial breast irradiation with high-dose-rate interstitial brachytherapy. Radiother Oncol 2009; 90: 43-47.

14. Vicini FA, Kestin LL, Edmundson GK et al. Dose-volume analysis for quality assurance of interstitial brachytherapy for breast cancer. Int Radiat Oncol Biol Phys 1999; 45: 803-810.

15. Kestin LL, Jaffray DA, Edmundson GK et al. Improving the dosimetric coverage of interstitial high dose rate breast implants. Int J Radiat Oncol Biol Phys 2000; 46: 35-43.

16. Das RK, Patel R, Shah H et al. 3D CT-based high-dose-rate breast brachytherapy implants: treatment planning and quality assurance. Int J Radiat Oncol Biol Phys 2004; 59: 1224-1228.

17. Wazer DE, Kramer B, Schmid C et al. Factors determine outcome in patients treated with interstitial implantation as a radiation boost for breast conservation therapy. Int J Radiat Oncol Biol Phys 1997; 39: 381-393.

18. Major T, Fröhlich G, Lovey K et al. Dosimetric experience with accelerated partial breast irradiation using image-guided interstitial brachytherapy. Radiother Oncol 2009; 90: 48-50.

19. Cholewka A, Szlag M, Ślosarek K et al. Comparison of 2Dand 3D-guided implantation in accelerated partial breast irradiation (APBI). J Contemp Brachytherapy 2009; 1: 207-210.

20. Tang J, Wee Tan P, Yangling Koh V et al. Multi-catheter interstitial accelerated partial breast irradiation - tips and tricks for a good insertion. J Contemp Brachytherapy 2014; 6: 85-90.

21. Gutiérrez C, Najjari D, Martínez E et al. The use of an interstitial boost in the conservative treatment of breast cancer: how to perform it routinely in radiotherapy department. J Contemp Brachytherapy 2015; 6: 397-403.

22. Skowronek J, Wawrzyniak-Hojczyk M, Ambrochowicz K. Brachytherapy in accelerated partial breast irradiation (APBI) - review of treatment methods. J Contemp Brachytherapy 2012; 4: $152-164$.

23. Gruszczyńska E, Dabkowski M, Kawczyńska M et al. Dosimetric comparison between 2D dose point and 3D CT-based volume optimization in breast boost HDR brachytherapy. World Congress of Brachytherapy, Barcelona, 2012 (Abstract).

24. Cholewka A, Szlag M, Białas B et al. The importance of the implant quality in APBI - Gliwice experience. Dosimetric evaluation. J Contemp Brachytherapy 2013; 5: 227-231.

25. Potter R, Van Limbergen E, Wambersie A. Reporting in brachytherapy: dose and volume specification. In: Gerbaulet A, Potter R, Mazeron JJ, Meertens H, Van Limbergen E (eds.). The GEC ESTRO Handbook of Brachytherapy. ESTRO, Brussels 2002; 155-215.

26. Shah Ch, Vicini F, Wazer D et al. The American Brachytherapy Society consensus statement for accreted partial breast irradiation. Brachytherapy 2013; 12: 267-277.

27. Strnad V, Hannoun-Levi JM, Guinot JL et al. Recommendations from GEC ESTRO Breast Cancer Working Group (I): Target definition and target delineation for accelerated or boost Partial Breast Irradiation using multicatheter interstitial brachytherapy after breast conserving closed cavity surgery. Radiother Oncol 2015; 115: 342-348.

28. Resch A, Pötter R, Van Limbergen E et al. Long-term results (10 years) of intensive breast conserving therapy including a high-dose and large-volume interstitial brachytherapy boost (LDR/HDR) for T1/T2 breast cancer. Radiother Oncol 2002; 63: 47-58.
29. Polgár C, Jánváry L, Major T et al. The role of high-dose-rate brachytherapy boost in breast conserving therapy: Longterm results of the Hungarian National Institute of Oncology. Rep Pract Oncol Radiother 2010; 15: 1-7. 